\title{
Integro-Differential Equations for a Jump-Diffusion Risk Process with Dependence between Claim Sizes and Claim Intervals
}

\author{
Heli Gao \\ Department of Mathematics, Binzhou University, Binzhou, China \\ Email: gaoheli81@163.com
}

How to cite this paper: Gao, H.L. (2016) Integro-Differential Equations for a JumpDiffusion Risk Process with Dependence between Claim Sizes and Claim Intervals. Journal of Applied Mathematics and Physics, 4, 2061-2068.

http://dx.doi.org/10.4236/jamp.2016.411205

Received: October 30, 2016

Accepted: November 19, 2016

Published: November 22, 2016

Copyright $\odot 2016$ by author and Scientific Research Publishing Inc. This work is licensed under the Creative Commons Attribution International License (CC BY 4.0).

http://creativecommons.org/licenses/by/4.0/

\begin{abstract}
The classical Poisson risk model in ruin theory assumed that the interarrival times between two successive claims are mutually independent, and the claim sizes and claim intervals are also mutually independent. In this paper, we modify the classical Poisson risk model to describe the surplus process of an insurance portfolio. We consider a jump-diffusion risk process compounded by a geometric Brownian motion, and assume that the claim sizes and claim intervals are dependent. Using the properties of conditional expectation, we establish integro-differential equations for the Gerber-Shiu function and the ultimate ruin probability.
\end{abstract}

\section{Keywords}

Jump-Diffusion Risk Process, Diffusion, Geometric Brownian Motion, Gerber-Shiu Function

\section{Introduction}

Various papers in ruin theory modify the classical Poisson risk model to describe the surplus process of an insurance portfolio. An extension of the classical model is that the risk process perturbed by a diffusion was first introduced by Gerber [1] and has been further studied by many authors during the last few years, e.g. Dufresne and Gerber [2], Gerber and Landry [3], Wang and Wu [4], Tsai and Willmot [5] [6], Chiu and Yin [7], and the references therein.

In the risk process that is perturbed by diffusion, the surplus process $U(t)$ of an insurance portfolio is given by 


$$
U(t)=u+c t-\sum_{i=1}^{N(t)} X_{i}+\sigma_{1} B_{1}(t), t \geq 0
$$

where $u \geq 0$ is the initial surplus, $c>0$ is the positive constant premium income rate, $S(t)=\sum_{i=1}^{N(t)} X_{i}$ is the aggregate claims process, in which

$$
N(t)=\sup \left\{k: T_{1}+T_{2}+\cdots+T_{k} \leq t\right\}
$$

is the claim number process (denoting the number of claims up to time $t$ ), and the interarrival times $\left\{T_{i}\right\}_{i=1}^{\infty}$ is a sequence of positive random variables. $\left\{X_{i}, i \geq 1\right\}$ is a sequence of nonnegative independent identically distributed (i.i.d.) random variables with distribution function $F(x)=1-\bar{F}(x)$ and density function $\mu=E(X),\left\{B_{1}(t), t \geq 0\right\}$ is a standard Brownian motion that is independent of the aggregate claims process $S(t), \sigma_{1}$ is a positive constant.

It is explicitly assumed in these papers that the interarrival times $\left\{T_{i}\right\}_{i=1}^{\infty}$ and the claim sizes $\left\{X_{i}, i \geq 1\right\}$ are mutually independent. However, this assumption is often too restrictive in practice, and there is a need for more general models where the independence assumptions can be relaxed. Recently, various results have been obtained concerning the asymptotic behavior of the probability of ruin for dependent claims, see [8]-[14], as well as the references therein. Zhao [14] assumed that the distribution of the time between two claim occurrences depends on the previous claim size. Motivated by the results of Zhao [14], the main aim of this paper is to modify the risk model (Equation (1)), and establish integro-differential equations for the Gerber-Shiu function and the ultimate ruin probability in the new risk model.

\section{Improved Risk Model}

In this paper, it is assumed that the claim occurrence process to be of the following type: If a claim $X_{i}$ is larger than a random variable $Y_{i}$, then the time until the next claim $T_{i+1}$ is exponentially distributed with rate $\lambda_{1}>0$, otherwise it is exponentially distributed with rate $\lambda_{2}>0$. The quantities $Y_{i}$ are assumed to be i.i.d. random variables with distribution function $G(x)$. Assuming that

$$
\mu<c\left[\frac{P(X>Y)}{\lambda_{1}}+\frac{P(X \leq Y)}{\lambda_{2}}\right],
$$

which is the net profit condition.

In the daily operation of insurance company, in addition to the premium income and claim to the operation of spending has a great influence on the outside, and there is also a factor that interest rates should not be neglected. As in [15], this paper assume that the risk model Equation (1) is invested in a stochastic interest process which is assumed to be a geometric Brownian motion $\left\{\mathrm{e}^{\Delta t}=\mathrm{e}^{r t+\sigma_{2} B_{2}(t)}\right\}$, where $r$ and $\sigma_{2}$ are positive constants, and $\left\{B_{2}(t), t \geq 0\right\}$ is a standard Brownian motion independent of $\{U(t), t \geq 0\}$. Let $X(t)$ denote the surplus of the insurer at time $t$ under this investment assumption. Thus, 


$$
X(t)=\mathrm{e}^{\Delta t}\left(u+\int_{0}^{t} \mathrm{e}^{-\Delta s} \mathrm{~d} U(s)\right), t \geq 0, X(0)=u .
$$

Denote $T$ to be the ruin time (the first time that the surplus becomes negative), i.e.,

$$
T=\inf \{t: X(t) \leq 0 \mid X(0)=u\},
$$

and $T=\infty$ if $X(t)>0, \forall t \geq 0$.

This article is interested in the expected discounted penalty (Gerber-Shiu) function:

$$
m_{\delta}(u)=E\left\{\mathrm{e}^{-\delta T} w[X(T-),|X(T)|] I(T<\infty) \mid X(0)=u\right\},
$$

where $I(\cdot)$ is the indicator function, $\delta>0$ is the force of interest and $w(x, y)$ is a nonnegative function of $x>0, y>0$ and satisfies $w(0,0)=1$.

Furthermore, let $T_{1}$ be the time when the first claim occurs, and random variable $T_{1 i}$ being exponentially distributed with rate $\lambda_{i}>0$. Assuming that

$$
P\left(T=T_{11}\right)=P(X>Y), \quad P\left(T=T_{12}\right)=P(X \leq Y) .
$$

For $i=1,2$, define

$$
m_{\delta(i)}(u)=E\left\{\mathrm{e}^{-\delta T} w[X(T-),|X(T)|] I(T<\infty) \mid X(0)=u, T=T_{1 i}\right\},
$$

such that

$$
m_{\delta}(u)=m_{\delta(1)}(u) P(X>Y)+m_{\delta(2)}(u) P(X \leq Y),
$$

then, $m_{\delta}(0)=m_{\delta(1)}(0)=m_{\delta(2)}(0)=1$.

\section{Integro-Differential Equations for $m_{\delta(i)}(u)$}

In this section, a system of integro-differential equations with initial value conditions satisfied by the Gerber-Shiu function $m_{\delta(i)}(u)$ is derived.

Define $a=r+\frac{\sigma_{2}^{2}}{2}$, and

$$
Y_{t}=\mathrm{e}^{\Delta t}\left(u+c \int_{0}^{t} \mathrm{e}^{-\Delta s} \mathrm{~d} s+\sigma_{1} \int_{0}^{t} \mathrm{e}^{-\Delta s} \mathrm{~d} B_{1}(s)\right)
$$

Lemma 3.1 Let $A_{t}=\sup _{s \in[0, t]}\left\{Y_{t} \vee 0\right\}$ for $t>0$. For $\varepsilon>0$ define the hitting time $T_{\varepsilon}=\inf \left\{t>0, Y_{t}-A_{t}=-\varepsilon\right\}$. Then, for $\alpha>0$, it can be concluded that

$$
E\left(\mathrm{e}^{-\alpha T_{\varepsilon}}, T_{\varepsilon}<\infty \mid X(0)=u\right)=1+o(\varepsilon), \varepsilon \rightarrow 0 .
$$

Proof $V_{t}:=Y_{t}-A_{t}$ is a reflecting diffusion with generator

$$
L f(x)=\frac{1}{2}\left(\sigma_{1}^{2}+\sigma_{2}^{2} x^{2}\right) \frac{\mathrm{d}^{2} f(x)}{\mathrm{d} x^{2}}+(c+a x) \frac{\mathrm{d} f(x)}{\mathrm{d} x},
$$

acting on functions satisfying the reflecting boundary condition $f^{\prime}(0)=0$.

If

$$
L f(x, t)+\frac{\partial f(x, t)}{\partial t}=0, x<0, t>0
$$


and $\left.\frac{\partial f(x, t)}{\partial x}\right|_{x=0}=0$ for $t>0$, then, according to Itô's formula $f\left(V_{t}, t\right)$ is a local martingale. Using the separation variable technique, we find that

$$
f(x, t)=\mathrm{e}^{-\alpha t} g(x) z(x)
$$

is a solution, where

$$
g(x)=\exp \left(\int_{x}^{o} \frac{c+a v}{\sigma_{1}^{2}+\sigma_{2}^{2} v^{2}} \mathrm{~d} v\right)
$$

$z(x)$ is a solution of

$$
z^{\prime \prime}(x)+r(x) z(x)=0 .
$$

Here

$$
r(x)=\frac{g^{\prime \prime}(x)\left(\sigma_{1}^{2}+\sigma_{2}^{2} x^{2}\right)+2(c+a x) g^{\prime}(x)-2 \alpha g(x)}{g(x)\left(\sigma_{1}^{2}+\sigma_{2}^{2} x^{2}\right)} .
$$

Using the initial condition $\left.\frac{\partial f(x, t)}{\partial x}\right|_{x=0}=0$ for $t>0$, we get $\frac{z^{\prime}(0)}{z(0)}=\frac{c}{\sigma_{1}^{2}}$, consequently

$$
\frac{z(0)}{z(\varepsilon)}=1+\frac{c}{\sigma_{1}^{2}} \varepsilon+o(\varepsilon)
$$

Applying the Optional Stopping Theorem, it follows that

$$
z(0)=E\left(\mathrm{e}^{-\alpha T_{\varepsilon}} g(-\varepsilon) z(-\varepsilon), T_{\varepsilon}<\infty \mid X(0)=u\right),
$$

and thus

$$
E\left(\mathrm{e}^{-\alpha T_{\varepsilon}}, T_{\varepsilon}<\infty \mid X(0)=u\right)=\frac{z(0)}{g(-\varepsilon) z(-\varepsilon)}=1+o(\varepsilon), \varepsilon \rightarrow 0
$$

This ends the proof of Lemma 3.1.

Similarly, the following lemma can also be obtained.

Lemma 3.2 Let $B_{t}=\sup _{s \in[0, t]}\left\{Y_{t} \wedge 0\right\}$ for $t>0$. For $\varepsilon>0$ define the hitting time $T_{\varepsilon}=\inf \left\{t>0, Y_{t}-A_{t}=\varepsilon\right\}$. Then, for $\alpha>0$, it can be concluded that

$$
E\left(\mathrm{e}^{-\alpha T_{\varepsilon}}, T_{\varepsilon}<\infty \mid X(0)=u\right)=1+o(\varepsilon), \varepsilon \rightarrow 0 .
$$

Theorem 3.1 Assuming that $m_{\delta(i)}(u)$ is second order continuously differentiable functions in $u$, then $m_{\delta(i)}(u)$ satisfies the following integro-differential equation

$$
\begin{aligned}
& \frac{1}{2}\left(\sigma_{1}^{2}+\sigma_{2}^{2} u^{2}\right) m_{\delta(1)}^{\prime \prime}(u)+(c+a u) m_{\delta(1)}^{\prime}(u)-\left(\lambda_{1}+\delta\right) m_{\delta(1)}(u) \\
& +\lambda_{1} \int_{0}^{u}\left[P(Y \leq y) m_{\delta(1)}(u-y)+P(Y>y) m_{\delta(2)}(u-y)\right] \mathrm{d} F(y), \\
& +\lambda_{1} \int_{u}^{+\infty} w(u, y-u) \mathrm{d} F(y)=0
\end{aligned}
$$




$$
\begin{aligned}
& \frac{1}{2}\left(\sigma_{1}^{2}+\sigma_{2}^{2} u^{2}\right) m_{\delta(2)}^{\prime \prime}(u)+(c+a u) m_{\delta(2)}^{\prime}(u)-\left(\lambda_{2}+\delta\right) m_{\delta(2)}(u) \\
& +\lambda_{2} \int_{0}^{u}\left[P(Y \leq y) m_{\delta(1)}(u-y)+P(Y>y) m_{\delta(2)}(u-y)\right] \mathrm{d} F(y), \\
& +\lambda_{2} \int_{u}^{+\infty} w(u, y-u) \mathrm{d} F(y)=0
\end{aligned}
$$

with the initial value conditions

$$
m_{\delta(1)}(0)=m_{\delta(2)}(0)=1, \quad m_{\delta(1)}^{\prime}(0)=m_{\delta(2)}^{\prime}(0)=0 .
$$

Proof Let $T_{11}$ be the time when the first claim occurs which exponentially distributed with rate $\lambda_{1}>0$. Consider the risk process $\{X(t): t \geq 0\}$ defined by Equation (2) in an infinitesimal time interval $(0, t)$. There are three possible cases in $(0, t)$ as follows.

1) There are no claims in $(0, t)$ with probability $1-\lambda_{1} t$, thus $X(t)=Y(t)$;

2) There is exactly one claim in $(0, t)$ with probability $\lambda_{1} t$. According to different of the claim amount, there are three possible cases in this case as follows.

a) The amount of the claim $y<Y(t)$, i.e., ruin does not occur, and thus $X(t)=Y(t)-y$;

b) The amount of the claim $y>Y(t)$, i.e., ruin occurs due to the claim;

c) The amount of the claim $y=Y(t)$, i.e., ruin occurs due to oscillation (observe that the probability that this case occurs is zero).

3) There is more than one claim in $(0, t)$ with probability $o(t)$.

Thus, considering the three cases above and noting that $\{X(t): t \geq 0\}$ is a strong Markov process, we have

$$
\begin{aligned}
m_{\delta(1)}(u)= & \mathrm{e}^{-\delta t}\left(1-\lambda_{1} t\right) E\left[m_{\delta(1)}\left(Y_{t}\right)\right]+\mathrm{e}^{-\delta t} \lambda_{1} t E\left[\int _ { 0 } ^ { Y _ { t } } \left(P(Y \leq y) m_{\delta(1)}\left(Y_{t}-y\right)\right.\right. \\
& \left.\left.+P(Y>y) m_{\delta(2)}\left(Y_{t}-y\right)\right) \mathrm{d} F(y)\right] \\
& +\mathrm{e}^{-\delta t} \lambda_{1} t \int_{Y_{t}}^{+\infty} w\left(Y_{t}, y-Y_{\mathrm{d} t}\right) \mathrm{d} F(y)+o(t)
\end{aligned}
$$

By Taylor expansion, we have $\mathrm{e}^{-\delta t}=1-\delta t+o(t)$, thus Equation (11) becomes

$$
\begin{aligned}
& \left(\lambda_{1}+\delta\right) t E\left[m_{\delta(1)}\left(Y_{t}\right)\right] \\
& =E\left[m_{\delta(1)}\left(Y_{t}\right)\right]-m_{\delta(1)}(u)+\mathrm{e}^{-\delta t} \lambda_{1} t E\left[\int _ { 0 } ^ { Y _ { t } } \left(P(Y \leq y) m_{\delta(1)}\left(Y_{t}-y\right)\right.\right. \\
& \left.\left.+P(Y>y) m_{\delta(2)}\left(Y_{t}-y\right)\right) \mathrm{d} F(y)\right] \\
& +\mathrm{e}^{-\delta t} \lambda_{1} t \int_{Y_{t}}^{+\infty} w\left(Y_{t}, y-Y_{\mathrm{d} t}\right) \mathrm{d} F(y)+o(t)
\end{aligned}
$$

Then, by Itô's formula we have

$$
\lim _{t \rightarrow 0} \frac{E\left[m_{\delta(1)}\left(Y_{t}\right)\right]-m_{\delta(1)}(u)}{t}=(c+a u) m_{\delta(1)}^{\prime}(u)+\frac{1}{2}\left(\sigma_{1}^{2}+\sigma_{2}^{2} u^{2}\right) m_{\delta(2)}^{\prime \prime}(u) .
$$

Therefore, by dividing $t$ on both sides of Equation (12), letting $t \rightarrow 0$, using Equation (13), we obtain Equation (9), and similarly we can obtain Equation (10).

The condition $m_{\delta(1)}(0)=m_{\delta(2)}(0)=1$ follows from the oscillating nature of the 
sample paths of $\{X(t)\}$. Now, we prove $m_{\delta(1)}^{\prime}(0)=m_{\delta(2)}^{\prime}(0)=0$.

For all $\varepsilon>0$, let $T=\inf \left\{t>0, Y_{t}-A_{t}=-\varepsilon\right\}, \tau=T \wedge T_{11}$. Then, by the strong property of $\{X(t)\}$, it can be concluded that

$$
\begin{aligned}
m_{\delta(1)}(0)= & E\left(\mathrm{e}^{-\delta \tau} m_{\delta(1)}(X(\tau) \mid X(0)=0)\right) \\
= & E\left(\mathrm{e}^{-\delta \tau} m_{\delta(1)}\left(X(\tau) I\left(T<T_{11}\right) \mid X(0)=0\right)\right) \\
& +E\left(\mathrm{e}^{-\delta \tau} m_{\delta(1)}\left(X(\tau) I\left(T>T_{11}\right) \mid X(0)=0\right)\right) \equiv I_{1}+I_{2}
\end{aligned}
$$

According to Lemma 3.1, it can be concluded that

$$
\begin{aligned}
I_{1} & =m_{\delta(1)}(0-\varepsilon) E\left(\mathrm{e}^{-\left(\alpha+\lambda_{1}\right) T}, T<\infty \mid X(0)=0\right)=m_{\delta(1)}(0-\varepsilon)+o(\varepsilon), \\
I_{2} & =\int_{0}^{+\infty} \int_{0}^{+\infty}\left[\lambda_{1} \mathrm{e}^{-\left(\alpha+\lambda_{1}\right) T} P(T>s \mid X(0)=0)+E\left(m_{\delta(1)}\left(Y_{s}-x\right) \mid X(0)=0\right)\right] \mathrm{d} s \mathrm{~d} F(x) \\
& =o(\varepsilon)
\end{aligned}
$$

Thus, $m_{\delta(1)}(0)=m_{\delta(1)}(0-\varepsilon)+o(\varepsilon)$, and correspondingly $m_{\delta(1)}^{\prime}(0-)=0$. Similar results can be derived for $m_{\delta(2)}^{\prime}(0-)$.

And for all $\varepsilon>0$, let $T^{\prime}=\inf \left\{t>0, Y_{t}-A_{t}=\varepsilon\right\}, \quad \tau^{\prime}=T^{\prime} \wedge T_{11}$, according to Lemma 3.2 we obtain $m_{\delta(1)}^{\prime}(0+)=m_{\delta(2)}^{\prime}(0+)=0$, thus $m_{\delta(1)}^{\prime}(0)=m_{\delta(2)}^{\prime}(0)=0$.

This ends the proof of Theorem 3.1.

\section{Differential Equations for $\Phi_{i}(u)$}

Let $\delta=0$ and $w(x, y) \equiv 1$ in Equation (3), correspondingly the expected discounted penalty function $m_{\delta}(u)$ turns into the ultimate ruin probability $\Phi_{i}(u)$.

Obviously,

$$
\Phi(u)=\Phi_{1}(u) P(X>Y)+\Phi_{2}(u) P(X \leq Y),
$$

and

$$
\Phi(0)=\Phi_{1}(0)=\Phi_{2}(0)=1
$$

Suppose that

$$
P(Y=0)=p, P(Y=u)=1-p \triangleq q,
$$

and $X_{i}$ is exponentially distributed with rate $\beta(\beta>0)$. Then, we get the following theorem.

Theorem 4.1 Assuming that $\Phi_{i}(u)$ is second order continuously differentiable functions in $u$, then $\Phi_{i}(u)$ satisfies the following integro-differential equation

$$
\begin{aligned}
& \frac{1}{2}\left(\sigma_{1}^{2}+\sigma_{2}^{2} u^{2}\right) \Phi_{1}^{\prime \prime \prime}(u)+\left[-\frac{1}{2} \beta \sigma_{2}^{2} u^{2}+\left(a+\sigma_{2}^{2}\right) u+\left(c-\frac{1}{2} \beta \sigma_{1}^{2}\right)\right] \Phi_{1}^{\prime \prime}(u) \\
& +\left[-\beta a u+\left(a-\lambda_{1}-c \beta\right)\right] \Phi_{1}^{\prime}(u)+q \lambda_{1} \beta \Phi_{1}(u)=q \lambda_{1} \beta \Phi_{2}(u) \\
& \frac{1}{2}\left(\sigma_{1}^{2}+\sigma_{2}^{2} u^{2}\right) \Phi_{2}^{\prime \prime \prime}(u)+\left[-\frac{1}{2} \beta \sigma_{2}^{2} u^{2}+\left(a+\sigma_{2}^{2}\right) u+\left(c-\frac{1}{2} \beta \sigma_{1}^{2}\right)\right] \Phi_{2}^{\prime \prime}(u) \\
& +\left[-\beta a u+\left(a-\lambda_{2}-c \beta\right)\right] \Phi_{2}^{\prime}(u)+p \lambda_{2} \beta \Phi_{2}(u)=p \lambda_{2} \beta \Phi_{1}(u)
\end{aligned}
$$


with the initial value conditions

$$
\Phi_{1}(0)=\Phi_{2}(0)=1, \Phi_{1}^{\prime}(0)=\Phi_{2}^{\prime}(0)=0 .
$$

Proof According to Equation (9), it can be concluded that

$$
\begin{aligned}
& \frac{1}{2}\left(\sigma_{1}^{2}+\sigma_{2}^{2} u^{2}\right) \Phi_{1}^{\prime \prime}(u)+(c+a u) \Phi_{1}^{\prime}(u)-\lambda_{1} \Phi_{1}^{\prime}(u) \\
& +\lambda_{1} \int_{0}^{u}\left[p \Phi_{1}(u-y)+q \Phi_{2}(u-y)\right] \beta \mathrm{e}^{-\beta y} \mathrm{~d} y+\lambda_{1} \mathrm{e}^{-\beta u}=0
\end{aligned}
$$

By taking the derivative with respect to $u$ on both sides of the above formula, and after some careful calculations, we obtain Equation (14). And similarly we can prove that Equation (15) holds. This ends the proof of Theorem 4.1.

\section{Conclusion}

In this paper, we consider a jump-diffusion risk process compounded by a geometric Brownian motion with dependence between claim sizes and claim intervals. We derive the integro-differential equations for the Gerber-Shiu functions and the ultimate ruin probability by using the martingale measure. Further studies are needed for the numerical solution of Equations (9), (10), (14) and (15). The results derived in this paper can be generalized to similar dependence ruin models.

\section{Acknowledgements}

This research was supported by the National Natural Science Foundation of China (No. 11601036), the Natural Science Foundation of Shandong (No. ZR2014GQ005) and the Natural Science Foundation of Binzhou University (No. 2016Y14).

\section{References}

[1] Gerber, H.U. (1970) An Extension of the Renewal Equation and Its Application in the Collective Theory of Risk. Skandinavisk Aktuarietidskrift, 1970, 205-210.

[2] Dufresne, F. and Gerber, H.U. (1991) Risk Theory for the Compound Poisson Process That Is Perturbed by Diffusion. Insurance: Mathematics and Economics, 10, 51-59. https://doi.org/10.1016/0167-6687(91)90023-Q

[3] Gerber, H.U. and Landry, B. (1998) On the Discounted Penalty at Ruin in a Jump-Diffusion and the Perpetual Put Option. Insurance: Mathematics and Economics, 22, 263-276. https://doi.org/10.1016/s0167-6687(98)00014-6

[4] Wang, G. and Wu, R. (2000) Some Distributions for the Classical Risk Process That Is Perturbed by Diffusion. Insurance: Mathematics and Economics, 26, 15-24. https://doi.org/10.2307/253675

[5] Tsai, C.C.L. and Willmot, G.E. (2002) A Generalized Defective Renewal Equation for the Surplus Process Perturbed by Diffusion. Insurance: Mathematics and Economics, 30, 51-66. https://doi.org/10.1016/s0167-6687(01)00096-8

[6] Tsai, C.C.L. and Willmot, G.E. (2002) On the Moments of the Surplus Process Perturbed by Diffusion. Insurance: Mathematics and Economics, 31, 327-350. https://doi.org/10.1016/s0167-6687(02)00159-2

[7] Chiu, S.N. and Yin, C.C. (2003) The Time of Ruin, the Surplus Prior to Ruin and the Deficit 
at Ruin for the Classical Risk Process Perturbed by Diffusion. Insurance: Mathematics and Economics, 33, 59-66. https://doi.org/10.1016/s0167-6687(03)00143-4

[8] Boudreault, M., Cossette, H., Landriault, D. and Marceau, E. (2006) On a Risk Model with Dependence between Interclaim Arrivals and Claim Sizes. Scandinavian Actuarial Journal, 5, 265-285. https://doi.org/10.1080/03461230600992266

[9] Bargès, M., Cossette, H., Loisel, S. and Marceau, E. (2011) On the Moments of the Aggregate Discounted Claims with Dependence Introduced by a FGM Copula. ASTIN Bulletin, 41, 215-238.

[10] Li, J. (2012) Asymptotics in a Time-Dependent Renewal Risk Model with Stochastic Return. Journal of Mathematical Analysis and Applications, 387, 1009-1023. https://doi.org/10.1016/j.jmaa.2011.10.012

[11] Yong, W. and Xiang, H. (2012) Differential Equations for Ruin Probability in a Special Risk Model with FGM Copula for the Claim Size and the Inter-Claim Time. Journal of Inequalities and Applications, 2012, 156. https://doi.org/10.1186/1029-242X-2012-156

[12] Fu, K.A. and Ng, C.Y.A. (2014) Asymptotics for the Ruin Probability of a Time-Dependent Renewal Risk Model with Geometric Lévy Process Investment Returns and DominatedlyVarying-Tailed Claims. Insurance Mathematics \& Economics, 56, 80-87. https://doi.org/10.1016/j.insmatheco.2014.04.001

[13] Zhang, J. and Xiao, Q. (2015 ) Optimal Investment of a Time-Dependent Renewal Risk Model with Stochastic Return. Journal of Inequalities and Applications, 2015,181. https://doi.org/10.1186/s13660-015-0707-3

[14] Zhao, X.H. and Yin, C.C. (2009) A Jump-Diffusion Risk Model with Dependence between Claim Sizes and Claim Intervals. Acta Mathematica Scientia, 29A, 1657-1664.

[15] Cai, J. and Xu, C. (2005) On the Decomposition of the Ruin Probability for a Jump-Diffusion Surplus Process Compounded by a Geometric Brownian Motion. North American Actuarial Journal, 10, 120-132. https://doi.org/10.1080/10920277.2006.10596255

\section{Submit or recommend next manuscript to SCIRP and we will provide best service} for you:

Accepting pre-submission inquiries through Email, Facebook, LinkedIn, Twitter, etc.

A wide selection of journals (inclusive of 9 subjects, more than 200 journals)

Providing 24-hour high-quality service

User-friendly online submission system

Fair and swift peer-review system

Efficient typesetting and proofreading procedure

Display of the result of downloads and visits, as well as the number of cited articles

Maximum dissemination of your research work

Submit your manuscript at: http://papersubmission.scirp.org/

Or contact jamp@scirp.org 